\title{
Characterization of the minimum domain required for targeting budding yeast myosin II to the site of cell division Ida MB Lister ${ }^{1,2}$, Nicola J Tolliday ${ }^{1,3}$ and Rong $\mathrm{Li}^{* 1,4}$
}

Address: ${ }^{1}$ Department of Cell Biology, Harvard Medical School, 240 Longwood Ave, Boston, MA, 02115, USA, ${ }^{2}$ Present address: Department of Molecular Biology and Microbiology, Tufts University School of Medicine, 136 Harrison Avenue, Boston, MA, 02111, USA, ${ }^{3}$ Present address: Broad Institute of Harvard and MIT, 7 Cambridge Center, Cambridge, MA 02142, USA and ${ }^{4}$ Present address: Stowers Institute for Medical Research, 1000 E. 50th Street, Kansas City, MO 64110, USA

Email: Ida MB Lister - ida.lister@tufts.edu; Nicola J Tolliday - tolliday@broad.harvard.edu; Rong Li* - rli@stowers-institute.org

* Corresponding author

Published: 26 June 2006

BMC Biology 2006, 4:19

doi:10.1186/1741-7007-4-19

This article is available from: http://www.biomedcentral.com/174I-7007/4/19

(C) 2006 Lister et al; licensee BioMed Central Ltd.

This is an Open Access article distributed under the terms of the Creative Commons Attribution License (http://creativecommons.org/licenses/by/2.0), which permits unrestricted use, distribution, and reproduction in any medium, provided the original work is properly cited.
Received: 20 April 2006

Accepted: 26 June 2006

\begin{abstract}
Background: All eukaryotes with the exception of plants use an actomyosin ring to generate a constriction force at the site of cell division (cleavage furrow) during mitosis and meiosis. The structure and filament forming abilities located in the C-terminal or tail region of one of the main components, myosin II, are important for localising the molecule to the contractile ring (CR) during cytokinesis. However, it remains poorly understood how myosin II is recruited to the site of cell division and how this recruitment relates to myosin filament assembly. Significant conservation between species of the components involved in cytokinesis, including those of the CR, allows the use of easily genetically manipulated organisms, such as budding yeast (Saccharomyces cerevisiae), in the study of cytokinesis. Budding yeast has a single myosin II protein, named Myol. Unlike most other class II myosins, the tail of Myol has an irregular coiled coil. In this report we use molecular genetics, biochemistry and live cell imaging to characterize the minimum localisation domain (MLD) of budding yeast Myol.
\end{abstract}

Results: We show that the MLD is a small region in the centre of the tail of Myol and that it is both necessary and sufficient for localisation of Myol to the yeast bud neck, the pre-determined site of cell division. Hydrodynamic measurements of the MLD, purified from bacteria or yeast, show that it is likely to exist as a trimer. We also examine the importance of a small region of low coiled coil forming probability within the MLD, which we call the hinge region. Removal of the hinge region prevents contraction of the CR. Using fluorescence recovery after photobleaching (FRAP), we show that GFP-tagged MLD is slightly more dynamic than the GFP-tagged full length molecule but less dynamic than the GFP-tagged Myol construct lacking the hinge region.

Conclusion: Our results define the intrinsic determinant for the localization of budding yeast myosin II and show it to be an oligomer of tentatively 3 monomers. We suggest that this is the minimum oligomeric unit (rather than the traditional myosin II dimer) that would allow specific assembly to the site of cytokinesis in a manner similar to the full length molecule. The flexible hinge region also contributes to CR structural integrity and contractility. 


\section{Background}

Budding yeast has one class II myosin, Myo1 [1,2], with the classic myosin superfamily domain organisation consisting of a conserved motor domain, a neck domain of 2 IQ motifs that binds the light chains Mlc1 and Mlc2 [3-5] and a tail region with a predicted coiled coil, as seen for all class II myosins so far studied. While the motor domain is responsible for the actin binding and ATP hydrolysis that allows the myosin to move along actin filaments, the class II myosin tails are responsible for how the motor function is used: the tails determine dimerisation, filament formation and localisation $[6,7]$.

There are two potential ways in which class II myosins may localise to the site of cell division: 1) by binding to other proteins, as for example in fission yeast, (Schizosaccharomyces pombe) where Mid1 is shown to temporally regulate the localisation of the major class II myosin, Myo2, [8]; 2) by filament formation, as shown in D. discoideum where filament formation is required for myosin II to localise to the cleavage furrow $[9,10]$. In non-muscle, smooth muscle and D. discoideum myosin II, it has also been shown that one or more small regions in the tail are responsible for filament formation $[7,9,11]$. Filament formation is thought to occur by the interaction of the charged residues (exposed by coiled coil formation) between two dimers. With the exception of fission yeast Myp2, which has a discontinuous coiled coil and forms a monomer [12], the class II myosins studied to date all form dimers. In $D$. discoideum, filament formation also depends on dephosphorylation of 3 serine residues $[7,9]$. It was also shown that dephosphorylation of Myo2 in fission yeast is necessary for localisation $[8,13]$. It should be noted that higher eukaryotic class II myosins are also regulated by phosphorylation of their light chains but this has not been demonstrated in $D$. discoideum or fission yeast

$[7,14]$.

Very little is known about the structure of budding yeast Myo1 and how it is recruited to the site of cell division. Unlike higher eukaryotic class II myosins, Myo1 is not predicted to consist of a continuous coiled coil as shown in the PAIR COIL [15] prediction plot in Fig. 1. This raises the question as to what kind of structure the Myo1 tail forms and how this might affect its cellular function and the structure of the contractile ring (CR). In order to determine if coiled coil formation is required for the localization of budding yeast myosin II, we set out to map the minimum domain in the tail region that is necessary and sufficient for Myo1 localisation. We use hydrodynamic measurements to assess its oligomeric nature and use FRAP analysis to compare the in vivo dynamics of the minimum localisation domain with the full length molecule. We also demonstrate that a region of low coiled coil form- ing potential that we have named the hinge region is important in the localisation and function of Myo1.

\section{Results}

A small region in the tail is sufficient for Myol localisation In order to determine whether there is a discrete region of Myo1 necessary and sufficient for localisation, deletion constructs were created and transformed into wild type and myoI $\Delta$ strains. The number of rings at the bud neck as a percentage of the total number of bud necks was calculated for each construct in both wild-type and $\Delta m y o 1$ strains and the results are shown in Fig 2, with fluorescent microscopy images of GFP labelled Myo1 and MLD shown below the localisation percentages. These results show that the minimum localisation domain (MLD) consists of residues 1044-1569. The coiled coil prediction plot shows that the MLD (indicated in Fig. 1) encompasses the region of low coiled coil forming probability, which includes 7 proline residues. Deletion of any additional parts of the MLD led to a lower percentage of localisation as compared to the full length MLD (Fig 2).

\section{The minimum localisation domain (MLD) of Myol forms an oligomer}

Since dimerisation and subsequently filament formation in many class II myosins is required for localisation we wanted to determine if the MLD forms oligomers. To this end, GST-MLD was purified after expression in E. coli. After removal of the GST tag, the MLD was applied to a Superose 6 gel filtration column. The MLD eluted at 49.4 $+/-0.4$ minutes $(\mathrm{n}=7)$ and the Stokes radius was obtained from a calibration curve of standards with known Stokes radii as shown in Fig. 3a; this resulted in a Stokes radius of $8.4+/-0.7 \mathrm{~nm}$ for the MLD (Fig. 3a and 3c). The void volume for the column was obtained using Dextran blue (2000 kDa, Stokes radius unknown), which gave an elution time of 32.2 minutes. Thyroglobulin, which has a molecular weight of $669 \mathrm{kDa}$ and an elution time of 49.0 minutes, ran close to the MLD, showing that the calibration curve was well within the upper resolution limit of the column. Purified MLD was also applied to a preformed 6-20\% sucrose density gradient and was found in fraction $7.6+/-0.5(n=4)$. A sedimentation coefficient obtained from a calibration curve of known sedimentation coefficients, as shown in Fig. 3b, was 5.2 +/- $0.3 \mathrm{~S}$ (n $=4)$. Neither gel filtration nor sucrose density gradient centrifugation can be used in isolation to obtain the molecular weight of the MLD as the presumed elongated nature of the protein will result in a larger apparent Stokes radius and a smaller apparent sedimentation coefficient as compared with a globular protein. This makes it difficult to determine the true molecular weight and thus the oligomeric nature of the MLD. However, combining the Stokes radius and the sedimentation coefficient in Equation 1 (see Materials and Methods) allowed us to deter- 


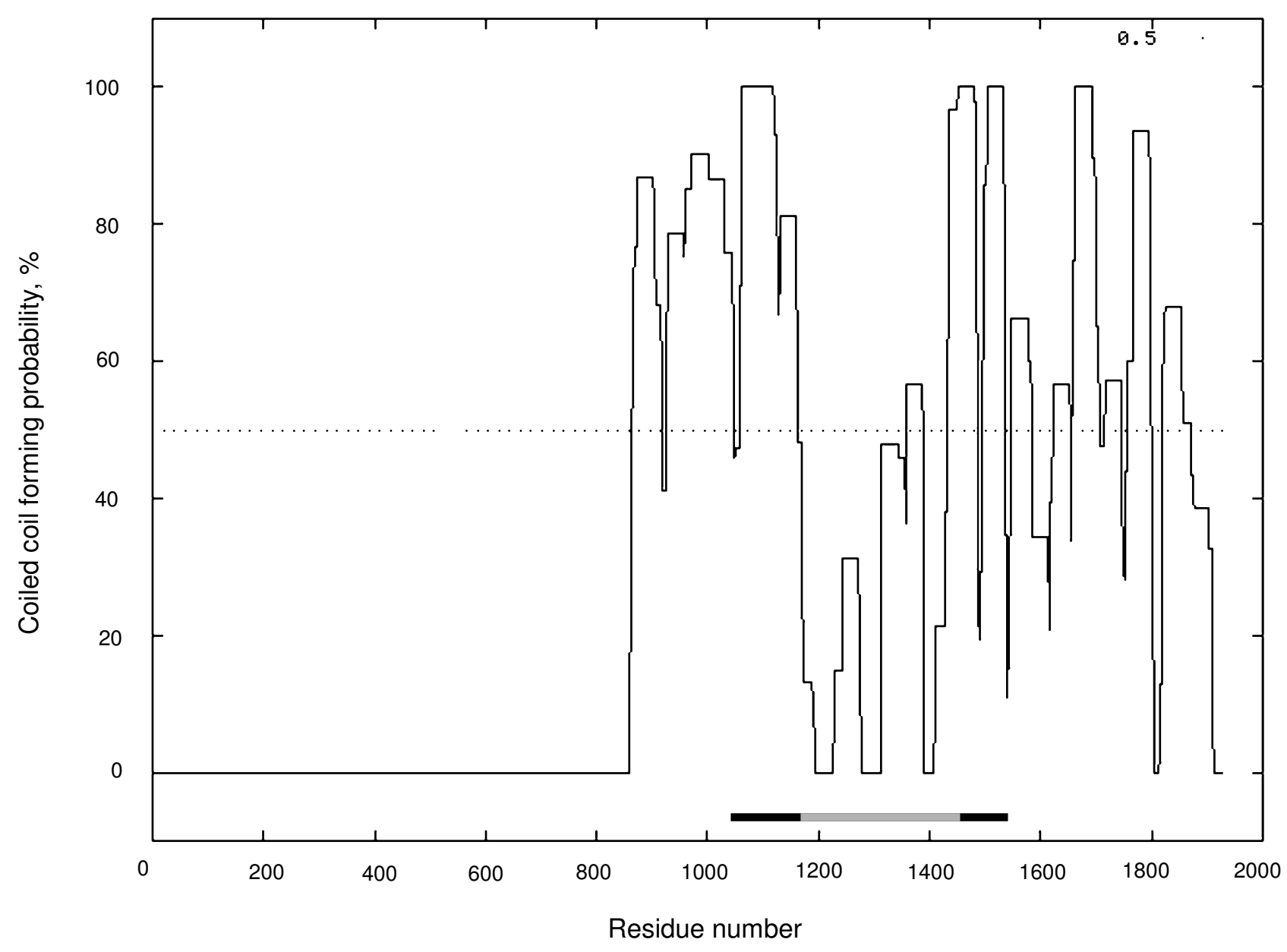

Figure I

Paircoil analysis of Myol. Coiled coil prediction of S. Cerevisiae Myol using the PAIRCOIL prediction program [I5]. The black bar denotes the position of the MLD and the gray bar within the black bar denotes the position of the hinge region within the MLD. The dotted line is shown as a reference point for $50 \%$ probability of coiled coil formation.

mine an experimental molecular weight of $177 \mathrm{kDa}$ for the MLD. When this value is divided by the calculated molecular weight for a monomer $(62 \mathrm{kDa})$, the oligomer is tentatively assigned to be a trimer (2.9 monomers).

We were concerned that the experimental values obtained were a product of expressing and purifying the MLD from E. coli. In order to be sure that our expressed protein was behaving in the same way as it would in yeast, the MLD with a C-terminal (myc) 6 tag was transformed into yeast (RLY 1232). Yeast extracts were prepared by grinding frozen cells under liquid nitrogen using a pestle and mortar; the powder was resuspended in a small volume of buffer and centrifuged to remove cellular debris and unlysed cells. $250 \mu$ lof the supernatant was applied to the Superose 6 gel filtration column. Fractions were collected and western blots were carried out after trichloroacetic acid
(TCA) precipitation to determine the elution position of MLD-(myc)6. As shown in Fig. 3c and 3d, MLD-(myc)6 eluted in the same fractions $(48.2+/-0.4$ minutes, $\mathrm{n}=4)$ as the expressed bacterial protein $(49.4+/-0.4$ minutes) indicating that they are hydrodynamically similar and thus that the trimer value assigned to MLD was not an artefact of expression in E. coli.

\section{Removing the MLD hinge region affects the function of Myol}

We observed that deletion of the MLD from Myo1 resulted in complete loss of localisation (Fig. 2) but that deletion of the hinge region (residues 1162-1430) within the MLD only lowered the efficiency of localisation to the bud neck to $70-80 \%$ of the full length molecule (Fig. 2) showing that the hinge region contributes to localisation but its loss does not abolish localisation. Since several 


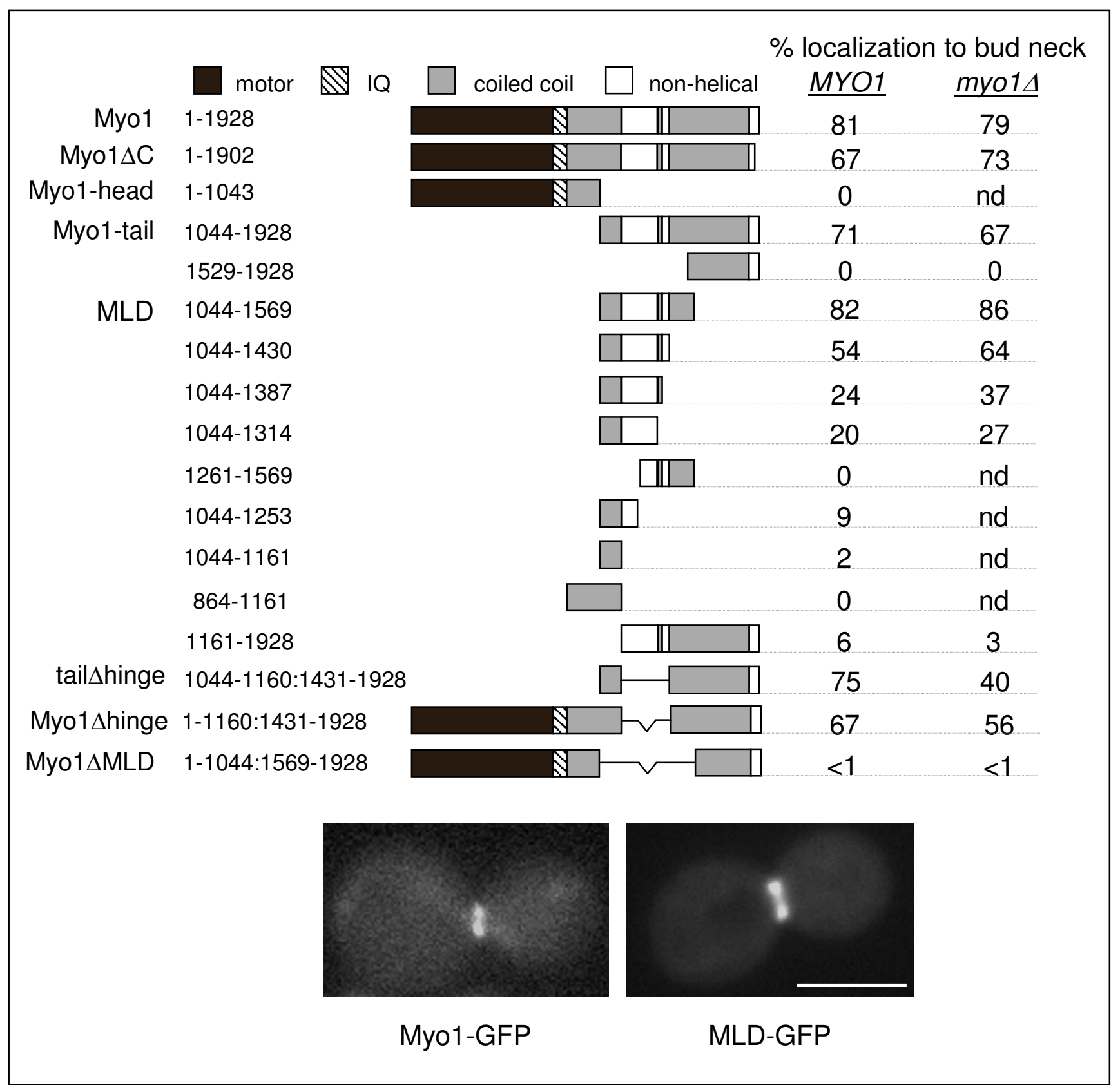

Figure 2

Localisation of Myol-GFP and deletion mutants in wild type and myol null yeast strains. Budding yeast strains (MYOI and myol $\Delta$ ) were transformed with Myol constructs tagged with GFP as shown here schematically. Fluorescence microscopy was used to quantify the number of fluorescent bud necks as a percentage of the total number of bud neck and these numbers are given on the right. Fluorescence microscopy images of budding yeast cells labeled with Myol-GFP and MLDGFP are shown below the localisation percentages. Scale bar denotes $5 \mu \mathrm{m}$.

other class II myosins contain kinks (where the coiled coil heptad repeat is interrupted) in their tail that are important for function, we wanted to examine the effect of removing the hinge region from Myo1 on CR contraction. A plasmid encoding Myo1 $\Delta$ hinge [Myo1(1-1161:1431-
1928)-GFP-(myc)6] under the control of the MYO1 promoter was transformed into the heterozygous diploid myo1s strain (RLY621), and haploid strains straining expressing Myo1 $\Delta$ hinge as the sole Myo1 were obtained by sporulation and tetrad dissection. 

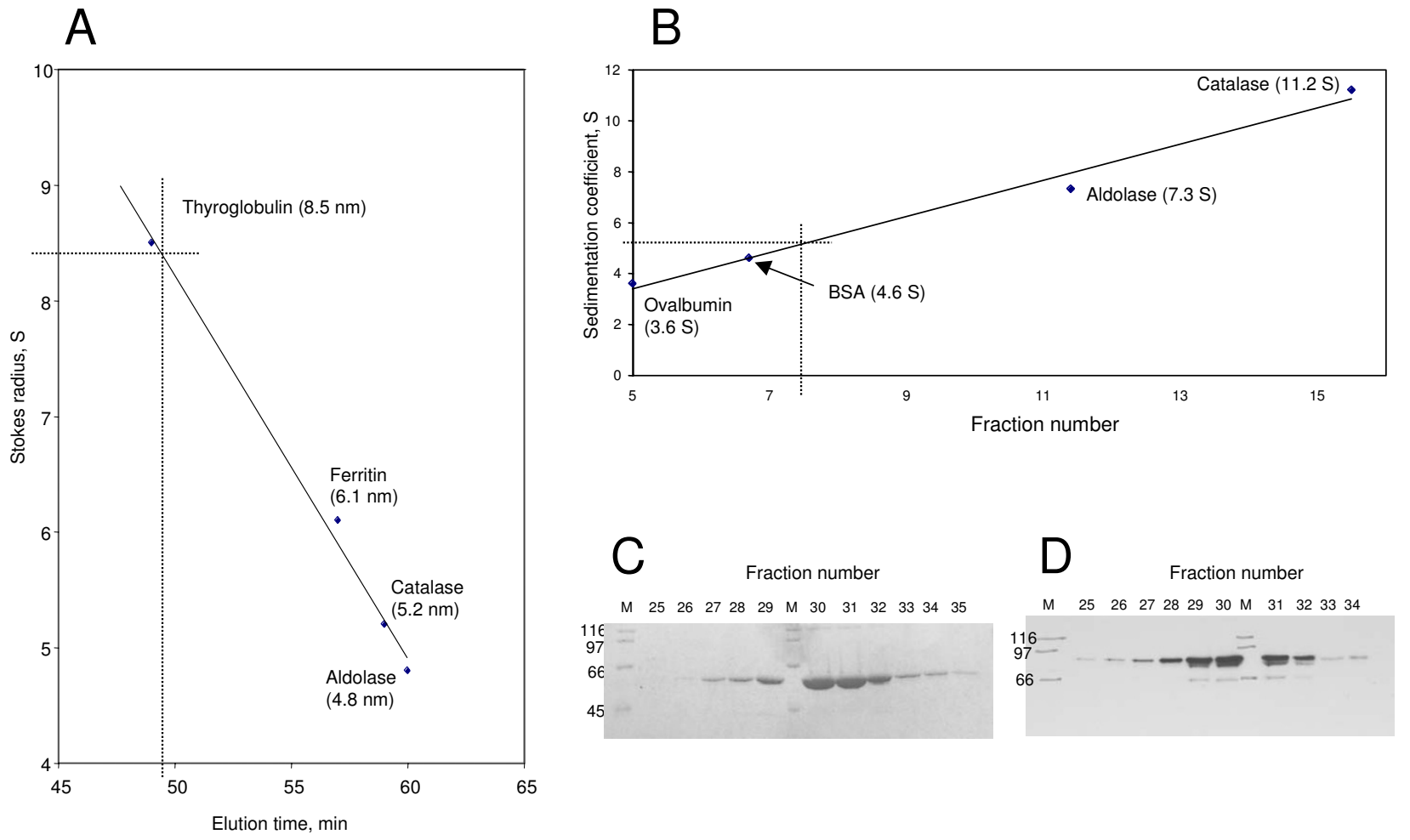

\section{Figure 3}

Hydrodynamic analysis of Myo I MLD A. Superose 6 gel filtration calibration curve with positions of protein standards of known Stokes radii shown. The dotted lines show the position of the Myol MLD with a measured Stokes radius of $8.4 \mathrm{~nm}$. B. Sucrose density gradient calibration curve with the positions of protein standards of known sedimentation coefficients shown. The dotted lines show the position of Myol MLD with a measured sedimentation coefficient of $5.2 \mathrm{~S}$. C. Coomassie Blue stained SDS-PAGE gel showing Superose 6 FPLC fractions of bacterially expressed MLD (after GST tag removal). Fractions were TCA precipitated before loading on the gel. Numbers to the left of the gel show molecular weight of markers (M). D. Western blot of Superose 6 FPLC fractions of MLD- $(\mathrm{myc}) 6$ yeast extract using anti-myc antibodies. Fractions were TCA precipitated before loading on the gel. Horizontal black marks and numbers to the left of the marks show molecular weight of markers (M).

We monitored contraction of the CR in wild-type and Myo1 $\Delta$ hinge strains. Cells were grown at the room temperature and arrested for $3 \mathrm{hrs}$ with nocodazole. The cells were then released from arrest and contraction was monitored using time-lapse confocal microscopy with images taken every $30 \mathrm{~s}$. Fig. 4 shows montages (A) and kymographs (B) of the wild-type and Myo1 $\Delta$ hinge ring as it goes through cytokinesis. Interestingly, Myo1 $\Delta$ hinge rings were unable to undergo contraction: the rings remained steadily at the bud neck until late in the cell cycle before gradually disappearing without reduction in diameter (n $=15)$. This was in contrast to the CR in wild-type cells, which contracted to a point before disappearing $(n=15)$.

\section{Fluorescence recovery after photobleaching (FRAP) studies of wild-type and mutant Myo I-GFP rings}

Fluorescence recovery after photobleaching (FRAP) enables one to examine the dynamics of a fluorescently 

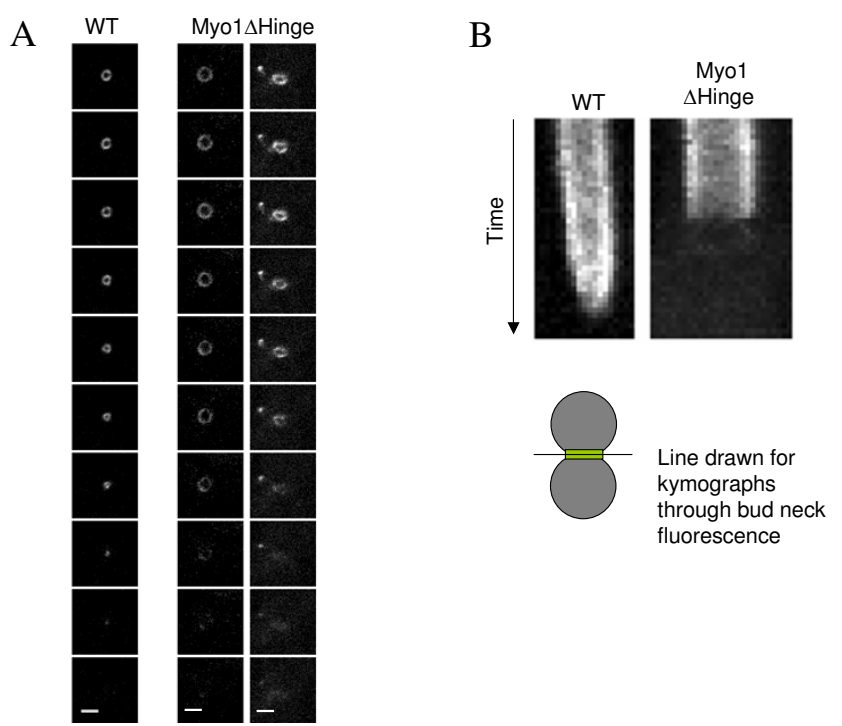

\section{Figure 4}

Time-lapse imaging of the wild-type and $\Delta$ hinge Myol ring contraction. Typical examples of Myol-GFP(myc)6 (RLYI33I) and Myol $\Delta$ hinge-GFP-(myc)6 (RLY2I39) $C R$ contraction as shown as a montage with images one minute apart (A) and as kymographs (B). Each horizontal line of the kymograph represents a time point of the image series with images taken at $30 \mathrm{~s}$ intervals, derived from a line drawn across the GFP signal at the bud neck (shown in the schematic below $B$ ). These lines are then stacked next to each other in chronological order to generate the kymograph. In A, scale bars denote $5 \mu \mathrm{m}$.

labelled molecular structure: fast recovery of the fluorescent signal indicates that there is a free pool of molecules that interchange rapidly with the molecules in the bleached area. We used FRAP to compare the in vivo dynamics of GFP-tagged full length Myo1, MLD and Myo1 $\Delta$ hinge [strains RLY1044, RLY1046 and RLY2139 (Table 2)]. Images were collected at 5 or 10 s intervals and analysed for fluorescence recovery. Table 1 shows the average percentage recovery and half time values. Myo1 $\Delta$ hinge appeared to be the most dynamic, recovering the greatest proportion of the bleached fluorescence and having the smallest recovery half time. This suggests that the contraction defect of the Myo1 $\Delta$ hinge could be due to a structural abnormality causing the mutant Myo1 $\Delta$ hinge molecules to be less well anchored within the CR. The MLD on the other hand appears to have a dynamic behaviour in between that of the full length molecule and Myo1 $\Delta$ hinge (Table 1) suggesting that the MLD is sufficient for stable association with the CR, but that other regions of Myo1 further stabilise the association.

\section{Discussion}

In the experiments described above, we have characterized the minimum domain required for targeting myosinII to the site of cell division in budding yeast. We show that this domain can oligomerise and form what appears to be trimers in solution and exhibits dynamics intermediate to those of the full length Myo1 molecule and Myo1 missing the region of low coiled coil forming probability (Myo1 $\Delta$ hinge)

How feasible is it that Myo1 might form a trimer? Coiled coils are a ubiquitous protein motif with the ability to form many different kinds of structures depending on the knobs-into-holes packing of the residues in the coiled coil and how this is disrupted by the distribution of breaks and stutters within the heptad repeat sequence [16]. The formation of triple stranded coiled coils is not unusual [16]; for example the influenza hemagglutinin protein [17] and the bacteriophage T4 whisker proteins [18] form parallel triple stranded coiled coils while the BAR domain of proteins such as amphiphysin consists of an antiparallel triple stranded coiled coil that dimerises [19]. Additionally trimers may be observed experimentally even when they have a dimeric coiled coil prediction $[20,21]$. Kammerer et al [21] provide evidence for a 5 residue trimerisation motif (R- [ILVM]-X-X- [ILV]-E) in short coiled coils. Interestingly two such motifs are present in the Myo1 tail (RVKSLE and RLSAVE at residues 1377-1382 and 1575-1580 respectively), one at the C-terminal end of the hinge region and one just beyond the C-terminal end of the MLD. Furthermore, many of the a and $\mathbf{d}$ residues of the heptad repeat in the Myo1 tail as predicted by either COILS [22,23] or PAIRCOIL [15] are asparginine, threonine and serine residues which can be prevalent in trimeric coiled coil structures [21].

In order to confirm the trimeric nature of the MLD, electron microscopy and analytical ultra centrifugation experiments were performed. Preliminary electron microscopy (EM) negative stain studies (in collaboration with D. Hanein and L. Hazelwood - results not shown) of purified MLD show a variety of oligomers which vary in length. Analytical ultra centrifugation experiments showed only protein aggregation indicating an inherent stickiness in the protein. Interestingly the elution peaks in gel filtration and sucrose density gradient were well defined and narrow (Figure $3 \mathrm{~b}$ and $3 \mathrm{c}$ ) indicating a single species of oligomer and also suggesting that the conditions necessary for EM or analytical centrifugation lead to protein instability. We speculate that the coiled coil domains present in the MLD are not long enough to cause stable association but that the MLD may be a minimal oligomerisation unit and possibly also a filament nucleation unit. That the MLD represents a normal assembly competent form is supported by the FRAP data which shows that 
Table I: FRAP total fluorescence recovery and half times

\begin{tabular}{lllll}
\hline Strain & Construct in $\Delta$ myol background & Percentage recovery (SD) & Half time (SD), s & Number of cells \\
\hline RLYI044 & Myol-GFP-6myc & $27(1 \mathrm{I})$ & $79(44)$ & 14 \\
RLYI046 & MLD-GFP-6myc & $40(14)$ & $50(38)$ & 11 \\
RLY2I39 & Myol hinge-GFP-6myc & $50(18)$ & $28(18)$ & 12 \\
\hline
\end{tabular}

the structure formed by the MLD was dynamically similar to that formed by full length Myo1 (Table 1). However further understanding of the nature and minumum oligomerisation unit (dimer or trimer) requires detailed structural and biochemical analysis of the native Myo1. If the trimeric nature suggested by our experiments of the MLD are indeed a property of the full length molecule, this would suggest a novel mode of contraction for the budding yeast CR as a Myo1 trimer would have 3 motor domains as compared with the two motor domains present in a more conventional dimeric structure.

As well as containing a region in the tail necessary for filament formation, all non-muscle myosins also contain tail sequences that disrupt the coiled coil formation and cause the tail to kink or bend sharply [7]. In higher eukaryotes bending at these sites is regulated by light chain phosphorylation, whereas in $D$. discoideum bending is regulated by heavy chain phosphorylation. Phosphorylation causes the tail to bend into a folded conformation that prevents filament formation [7]. We have shown that the region of low coiled coil forming probability in Myo1, termed the hinge, is important for CR contraction. The FRAP data also show that fluorescence recovery of Myo1 $\Delta$ hinge is faster than that of the wild-type molecule. It is interesting that the MLD, which possesses the hinge region, has a slower exchange half time than Myo1 $\Delta$ hinge, suggesting that the hinge has a role in anchoring Myo1 in the CR. Taken together these data suggest that removal of the hinge prevents proper CR assembly, resulting in reduced structural stability. The inability of the hingeless CR to contract could either be due to a lack of force generation or due to rapid disassembly when under force. Interestingly, Lord et al [24] have recently shown that the motor domain of Myo1 is not necessary for contraction to occur at the bud neck during cytokinesis in budding yeast, though the rate of contraction is slower than wild type. This suggests that there is an additional force generating element in the budding yeast CR and that the Myo1 tail may serve to guide this force. The hinge region may play a direct role in linking the two force generating elements. The additional force generating element acting alone in the absence of Myo1 or in the presence of a defective Myo 1 could come from Chs2, the enzyme that synthesizes and deposits chitin during cytokinesis and this synthetic energy could be used to drive membrane closure $[1,25,26]$. Normally this force would be coupled with the force generated by Myo1 and we speculate that the tail of Myo1 may interact directly with Chs2. Future studies will examine the localisation of other bud neck components in the presence of the MLD or the hingeless Myo1 to see if their localisation is perturbed. This will further our understanding of the cytokinetic defects caused by the loss of hinge in Myo1 and of how it interacts with other bud neck components.

Finally, how does the MLD compare with the localisation determinants of other class II myosins? In fission yeast Myo2, the region required for localization is different: it was shown that a small C-terminal domain is necessary and sufficient for localisation to the contractile ring $[8,13]$. Phosphorylation plays a regulatory role in Myo2 localisation, though there is disagreement over the exact residues involved. There is also evidence that the very $\mathrm{C}$ terminus of budding yeast Myo1 is phosphorylated [27], but the precise residues and function of this phosphorylation are not known. Our results showing localisation in a Myo1 construct lacking the C-terminus (Myo1 1-1908, Fig. 2) suggest that this phosphorylation would not be required for Myo1 localisation.

It remains open whether myosin-II recruitment is mediated by a specific interaction, with certain components of the cell division site, or by an interaction that requires certain structural features of the myosin-II complex assembly. Regardless, the MLD can be used as bait for the identification of the binding partners responsible for the recruitment of myosin-II to the bud neck. However, given the potential requirement for structural determinants and the fact that direct recruiting factors for myosin-II have yet to be found, we speculate that recruitment of myosin-II may be mediated by multiple low affinity interactions that rely on structural assembly of the CR and may not be detectable by conventional solution-based assays for protein interactions.

\section{Conclusion}

Our results show that a small defined region in tail of budding yeast myosin II, the MLD, is necessary and sufficient for localisation. We show that when expressed in bacteria or in yeast, this domain behaves like a trimer rather than like the more traditional class II myosin dimer, raising the possibility that if the trimeric nature holds for the full length Myo1, the structure of the CR in budding yeast 
Table 2: Strains

\begin{tabular}{|c|c|c|c|}
\hline Name & Genotype & Background & Source \\
\hline RLY26I & MATa ura3-I his3-II, I 5 leu2-3, I I 2 trpl ade2-I barl $\Delta$ & W303a & Elion Lab \\
\hline RLY62I & MATal $\alpha$ ura3/ura3 his3/his3 leu2/leu2 trp I/trp I myo I $4:: T R P I / M Y O I$ & $\mathrm{~S} 288 \mathrm{c}$ & Li Lab \\
\hline RLY622 & MAT $\alpha$ ura3 his3 leu2 trp I myo I A::TRPI & $\mathrm{S} 288 \mathrm{c}$ & Li Lab \\
\hline RLY75I & MATa ura3-I his3-I I, I leu2-3, I I 2 trpl-I ade2-I barl A pMYOI(I044-1928)-GFP:LEU2 (pNTI3) & W303a & This work \\
\hline RLYI034 & MATa ura3-I his3-II, I5 leu2-3,II 2 trp I-I ade2-I barl $\triangle$ pMYOI(I044-I569)-GFP-6myc:LEU2 (pNT49) & W303a & This work \\
\hline RLYI035 & MATa ura3-I his3-II,I5 leu2-3, I I 2 trp I-I ade2-I barl $\triangle$ pMYOI-GFP-6myc:LEU2 (pNT50) & W303a & This work \\
\hline RLYI040 & $\begin{array}{l}\text { MATal } \alpha \text { ura3-I/ura3-I his3-I I, I 5/his3-I I, I } 5 \text { leu2-3, I I /leu2-3, I I } 2 \text { trp I-I/trp I-I ade2-I/ade2-I barl } 4 / \text { barl } \Delta \\
\text { myo I A::TRPIMYO I }\end{array}$ & W303a & This work \\
\hline RLYI044 & MAT $\alpha$ ura3 his3 leu2 trp I myo I A::TRPI pMYOI-GFP-6myc:LEU2 (pNT50) & S288c & This work \\
\hline RLYI046 & MAT $\alpha$ ura3 his3 leu2 trp I myo I A::TRPI pMYOI(I044-1569)-GFP-6myc:LEU2 (pNT49) & $\mathrm{S} 288 \mathrm{c}$ & This work \\
\hline RLYI05I & MATa ura3-I his3-I I, I 5 leu2-3, I I 2 trp I-I ade2-I barl $\triangle$ pMYOI(I-I043)-GFP-6myc:LEU2 (pNT5 I) & W303a & This work \\
\hline RLYI073 & MATa ura3-I his3-II,I5 leu2-3,II 2 trp I-I ade2-I barl $\triangle$ pMYOI(I044-I 253)-GFP-6myc:LEU2 (pNT62) & W303a & This work \\
\hline RLYI074 & MATa ura3-I his3-I I, I 5 leu2-3, I I 2 trp I-I ade2-I barl $\triangle$ pMYOI(I 26I-I569)-GFP-6myc:LEU2 (pNT63) & W303a & This work \\
\hline RLYI096 & $\begin{array}{l}\text { MATal } \alpha \text { ura3-I/ura3-I his3-II,15/his3-I I, I } 5 \text { leu2-3, I I /leu2-3, I I } 2 \text { trp I-I/trp I-I ade2-I/ade2-I barl A/barl } \Delta \\
\text { myo I A::TRPIMYOI pMYOI(I-1902)-GFP-6myc:LEU2 (pNT66) }\end{array}$ & W303a & This work \\
\hline RLYIIOI & MATa ura3-I his3-II, I5 leu2-3, I I 2 trp I-I ade2-I barl $\triangle$ pMYOI(I-1902)-GFP-6myc:LEU2 (pNT66) & W303a & This work \\
\hline RLYIIO3 & $\begin{array}{l}\text { MATa ura3-I his3-II, I5 leu2-3, I I } 2 \text { trpl-I ade2-I barl } \triangle \text { myol A*:TRP pMYOI(I-I902)-GFP-6myc:LEU2 } \\
\text { (pNT66) }\end{array}$ & W303a & This work \\
\hline RLYIII9 & MATa ura3-I his3-I I, I leu2-3,II 2 trp I-I ade2-I barl $\triangle$ pMYOI(I044-II6I)-GFP-6myc:LEU2 (pNT7I) & W303a & This work \\
\hline RLYI212 & MAT $\alpha$ ura3 his3 leu2 trp I myol A::TRPI pMYOI(I044-1928)-GFP-6myc:LEU2 (pNT76) & S288c & This work \\
\hline RLYI288 & MATa ura3-I his3-II,I5 leu2-3,II 2 trp I-I ade2-I barl $\triangle$ pMYOI(II6I-1928)-GFP-6myc:LEU2 (pNT94) & W303a & This work \\
\hline RLYI295 & MATa ura3-I his3-II, I leu2-3, I I2 trp I-I ade2-I barl $\triangle$ pMYOI(864-I I6I)-GFP-6myc:LEU2 (pNT96) & W303a & This work \\
\hline RLYI295 & MATa ura3-I his3-II, I5 leu2-3,I I 2 trpI-I ade2-I barl $\triangle$ pMYOI(864-I I 6I)-GFP-6myc:LEU2 (pNT96) & W303a & This work \\
\hline RLYI297 & MATa ura3-I his3-I I,I 5 leu2-3,I I 2 trpI-I ade2-I barl $\triangle$ pMYOI(I529-1928)-GFP:LEU2 (pNT40) & W303a & This work \\
\hline RLYI298 & MAT $\alpha$ ura3 his3 leu2 trp I myo I A::TRPI pMYOI(I529-1928)-GFP:LEU2 (pNT40) & $\mathrm{S} 288 \mathrm{c}$ & This work \\
\hline RLYI307 & MATa ura3-I his3-I I, I leu2-3,I I 2 trp I-I ade2-I barl $\triangle$ pMYOI(I044-I430)-GFP-6myc:LEU2 (pNTI I0) & W303a & This work \\
\hline RLYI308 & MATa ura3-I his3-I I, I5 leu2-3, I I 2 trp I-I ade2-I barl $\triangle$ pMYOI(I044-1387)-GFP-6myc:LEU2 (pNTIII) & W303a & This work \\
\hline RLYI309 & MATa ura3-I his3-I I, I 5 leu2-3, I I 2 trp I-I ade2-I barl A pMYOI(I044-I3 I 4)-GFP-6myc:LEU2 (pNTI I 2) & W303a & This work \\
\hline RLYI3I0 & MAT $\alpha$ ura3 his3 leu2 trp I myo I A::TRPI pMYOI(I044-I 430)-GFP-6myc:LEU2 (pNTI I0) & $\mathrm{S} 288 \mathrm{c}$ & This work \\
\hline RLYI3II & MAT $\alpha$ ura3 his3 leu2 trpI myo I A::TRPI pMYOI(I044-I 387)-GFP-6myc:LEU2 (pNTIII) & $\mathrm{S} 288 \mathrm{c}$ & This work \\
\hline RLYI3/2 & MAT $\alpha$ ura3 his3 leu2 trp I myo I A::TRPI pMYOI(I044-13 I 4)-GFP-6myc:LEU2 (pNTI I 2 & $\mathrm{~S} 288 \mathrm{c}$ & This work \\
\hline RLYI330 & $\begin{array}{l}\text { MATal } \alpha \text { ura3-I/ura3-I his3-I I,I 5/his3-II, I } 5 \text { leu2-3, I I I/leu2-3, I I } 2 \text { trp I-I/trp I-I ade2-I/ade2-I barl } \Delta \\
\text { myo I A::KANIMYOI pMYOI-GFP-6myc:LEU2 (pNTI I I) }\end{array}$ & W303a & This work \\
\hline RLYI33I & MAI $\alpha$ ura3-I his3-II leu2-3, I I 2 trp I-I ade2-I barl $\triangle$ myo I $\triangle:$ KAN pMYOI-GFP-6myc:LEU2 (pNTI I 3) & W303a & This work \\
\hline RLYI577 & $\begin{array}{l}\text { MATa ura3-I his3-II,I5 leu2-3,III trpI-I ade2-I barl } 1 \text { pMYOI(I044-II 60:I43I-1928)-GFP-6myc:LEU2 } \\
\text { (pNTI2I) }\end{array}$ & W303a & This work \\
\hline RLYI578 & MAT $\alpha$ ura3 his3 leu2 trp I myo I A::TRPI pMYOI(I044-I I60:143I-1928)-GFP-6myc:LEU2 (pNTI2I) & $\mathrm{S} 288 \mathrm{c}$ & This work \\
\hline RLYI59I & MAT $\alpha$ ura3 his3 leu2 trp I myo I A::TRPI pMYOI(I I6I-1928)-GFP-6myc:LEU2 (pNT94) & S288c & This work \\
\hline RLYI598 & MATa ura3-I his3-I I, I5 leu2-3, I I 2 trp I-I ade2-I barl $\triangle$ myo I A::TRP pMYOI-GFP-6myc:URA3 (pNTII3) & W303a & This work \\
\hline RLYI653 & $\begin{array}{l}\text { MATa ura3-I his3-I I, I } 5 \text { leu2-3, I I } 2 \text { trp I-I ade2-I barl } \triangle \text { myol A::TRP pMYO I-GFP-6myc:URA3 (pNTI I3) } \\
\text { p:LEU2 (pRL304) }\end{array}$ & W303a & This work \\
\hline RLYI655 & $\begin{array}{l}\text { MATa ura3-I his3-II, I5 leu2-3, I I } 2 \text { trp I-I ade2-I barl } \triangle \text { myo I A::TRP pMYOI-GFP-6myc:URA3 (pNTII3) g } \\
\text { pMYOI-GFP-6myc:LEU2 (pNT50) }\end{array}$ & W303a & This work \\
\hline RLYI657 & $\begin{array}{l}\text { MATa ura3-I his3-I I, I5 leu2-3, I I } 2 \text { trp I-I ade2-I bar I } \triangle \text { myo I } 4: \text { KAN pMYO I-GFP-6myc:URA3 (pNTI I 3) } \\
\text { pMYOI(I-I045:I 570-1928)-GFP-6myc:LEU2 (pNTI 20) }\end{array}$ & W303a & This work \\
\hline RLYI659 & $\begin{array}{l}\text { MATa ura3-I his3-II, I5 leu2-3,II } 2 \text { trp I-I ade2-I barl } \triangle \text { pMYOI(I-I045:I570-1928)-GFP-6myc:LEU2 } \\
\text { (pNTI20) }\end{array}$ & W303a & This work \\
\hline RLYI660 & MAT $\alpha$ ura3 his3 leu2 trpI myo I A::TRPIg pMYOI(I-1045:I570-1928)-GFP-6myc:LEU2 (pNTI20) & S288c & This work \\
\hline RLY2I 27 & MAT $\alpha$ ura3 his3 leu2 trp I myo I A::TRP, pMYOI(I-I I60:I43 I-1928)-GFP-6myc:LEU (pILII) & $\mathrm{S} 288 \mathrm{c}$ & This work \\
\hline RLY2I 28 & MATa ura3-I his3-II, I5 leu2-3, I I 2 trpI-I ade2-I barl $\triangle$ pMYOI(I-I I 60:I43 I-I928-GFP-6myc):LEU (pILII) & W303a & This work \\
\hline RLY2I 32 & MAT $\alpha$ ura3 his3 leu2 trp I MLD-6myc:LEU (pILI3) & $\mathrm{S} 288 \mathrm{c}$ & This work \\
\hline RLY2139 & MATa ura3 his3 leu2 trp I myo I A::TRPI pMYOI(I-I | 60:I43I-1928) -GFP-6myc:LEU (pILII) & S288c & This work \\
\hline
\end{tabular}

might be different than were it composed in part of a dimeric myosin II. The similarity between the dynamics of the MLD and full length budding yeast myosin II in vivo as explored through our FRAP studies lends confidence that the MLD assembly in vivo is structurally similar to that of full length Myo1. We also show that the MLD encompasses a region of low coiled coil forming probability (the hinge) which, when removed, does not abolish myosin II localisation to the bud neck, the site of cell division, but does prevent proper contraction of the CR during cytoki- 
nesis. We propose that this hinge region may interact directly with other components of the CR and that its loss leads to a more dynamic, less anchored molecule as shown by the faster fluorescent turnover seen in our FRAP studies in comparison with the MLD and full length protein.

\section{Methods \\ Media and genetic manipulations}

Yeast cell culture and genetic techniques were carried out by methods described in [28]. Yeast extract, peptone, dextrose (YPD) contained 2\% glucose, 1\% yeast extract and 2\% Bactopeptone (Difco Laboratories, Detroit, MI). Synthetic complete (SC) media was prepared by the method described [29].

\section{Plasmid construction}

The plasmid expressing Myo1-GFP-(myc)6 (pNT50) was generated by excising MYO1 from a previously-described plasmid encoding Myo1-GFP (pLP8 [30]) with PstI and BamHI, and ligating it into PstI-BamHI-digested pRL304, a pRS315-based vector for C-terminal tagging with a GFP(myc)6 fusion. To generate Myo1-GFP-(myc)6 in a pRS316 (URA3)-based vector, the $7 \mathrm{~kb}$ fragment from SalI-NotI digested pNT50 was ligated to SalI-NotI digested pRS316, creating pNT113. To generate the plasmid that expresses the C-terminal 884 amino acids of Myo1p under the control of the MYO1 promoter with a C-terminal GFP tag (pNT13), the sequence encoding the promoter region and the first 1043 amino acids was excised from pLP8, using PstI and BglII, and replaced with just the promoter region (generated by PCR and digested with PstI and $B g l$ II). The GFP tag of pNT13 was replaced with a GFP(myc)6 fusion by ligating the $1 \mathrm{~kb}$ fragment generated by BamHI-NotI digest of pNT50 to BamHI-NotI digested pNT13, creating pNT76. To create the plasmid expressing Myo1(1044-1569), pNT13 was double digested with BglII and HaeIII yielding a $1.6 \mathrm{~kb}$ fragment encoding Myo1(1044-1569). pNT13 was digested with BamHI, blunted and digested with $B g l \mathrm{II}$, to yield a $7 \mathrm{~kb}$ fragment encoding the vector backbone, MYO1 promoter, and GFP. Ligation of both fragments produced an in-frame fusion of Myo1(1044-1569) and GFP (pNT43), which was then excised using Pst I and BamHI, and ligated into PstIBamHI-digested pRL304 to create Myo1(1044-1569)GFP-(myc)6 (pNT49). The sequence encoding Myo1(1529-1928) was generated by PCR from yeast genomic DNA and digested with BglII and BamHI (restriction sites contained within the forward and reverse primers, respectively), and ligated to the $6.9 \mathrm{~kb}$ fragment produced by BglII-BamHI double digest of pNT13, to generate Myo1(1529-1928)-GFP (pNT40). To create the plasmid expressing Myo1(1-1043), pNT50 was doubledigested with $B g l \mathrm{II}$ and BamHI, blunted and self-ligated yielding an in-frame fusion of Myo1(1-1043) and GFP- (myc)6 (pNT51). The sequence encoding Myo1(10441253 ) was generated by digesting pNT43 with XbaI, blunting, and digesting with Pst $\mathrm{I}$, yielding a $0.9 \mathrm{~kb}$ fragment. This fragment was ligated to pNT49 that had been digested with Bam HI, blunted, and digested with PstI, to create Myo1(1044-1253)-GFP-(myc)6 (pNT62). pNT43 was digested with $\mathrm{XbaI}$, blunted and digested with BamHI to produce a $0.9 \mathrm{~kb}$ fragment encoding Myo1(12611569). This was ligated to pNT49 that had been digested with BglII, blunted, and digested with BamHI, to create Myo1(1261-1569)-GFP-(myc)6 (pNT63). A construct expressing Myo1 lacking the C-terminal 26 amino acids was generated by utilizing an existing EcoRI site, as described previously [27]. Briefly, MYO1, cloned PstIBam HI in the Bluescript vector (pLP9), was digested with EcoRI to yield a $6 \mathrm{~kb}$ fragment encoding Myo1(1-1902). This fragment was ligated to EcoRI-digested pRL164, a pRS313-based vector for C-terminal GFP tagging, to generate pRL221. This plasmid was double-digested with $B g l I I$ and BamHI to generate a fragment encoding Myo1(1044-1902), and this was ligated to BglII-BamHI digested pNT50 to create Myo1(1-1902)-GFP-(myc)6 (pNT66). The sequence encoding Myo1(1044-1161) was generated by PCR from yeast genomic DNA and digested with $B g l \mathrm{II}$ and BamHI (restriction sites contained within the forward and reverse primers, respectively) and ligated to the $6.9 \mathrm{~kb}$ fragment produced by BglII-BamHI double digest of pNT13 to generate Myo1(1044-1163)-GFP (pNT69). The GFP tag of pNT69 was replaced with a GFP(myc) 6 fusion by ligating the $1 \mathrm{~kb}$ fragment generated by BamHI-NotI digest of pRL304 to BamHI-NotI digested pNT69, creating pNT71. The sequences encoding Myo1(1160-1928), Myo1(1044-1430), Myo1(10441387) and Myo1(1044-1314) were generated by PCR from yeast genomic DNA, digested with BglII and BamHI (restriction sites contained within the forward and reverse primers, respectively) and ligated to the $7.3 \mathrm{~kb}$ fragment produced by BglII-BamHI double digest of pNT76, to generate pNT94, pNT110, pNT111 and pNT112, respectively. Similarly, the sequence encoding Myo1(864-1161) was generated by PCR from yeast genomic DNA, digested with BamHI (restriction site contained within the forward and reverse primers) and ligated to the $7.3 \mathrm{~kb}$ fragment produced by BglII-Bam HI double digest of pNT76, to generate Myo1(864-1161)-GFP-(myc)6 (pNT96). To create a deletion of the non-helical sequences within Myo1-tail, pNT121 was constructed. Briefly, the sequence encoding Myo1(1431-1928) was generated by PCR from yeast genomic DNA, digested with BamHI (restriction site contained within the forward and reverse primers) and ligated to BamHI digested pNT71, to generate Myo1(1044-1160:1431-1928)-GFP-(myc)6. Similarly, the sequence encoding Myo1(1570-1928) was generated by PCR from yeast genomic DNA, digested with BglII and BamHI (restriction sites contained within the forward and 
reverse primers, respectively) and ligated to BglII-BamHI digested pNT50, to generate Myo1(1-1044:1670-1928)GFP-(myc)6 (pNT120). To create a deletion of the nonhelical region of the MLD in the full length Myo1, pIL11 was constructed. Briefly pNT121 was cut with PstI/BglII to yield a 7000 bp fragment encoding Myo1(10441161:1439-1928). This fragment was ligated with a 3300 bp fragment encoding Myo1 promoter -Myo1(11043) cut with PstI/BglII from pLP8. To create a plasmid (pIL10) encoding N-terminally tagged GST-MLD, a PCR product encoding Myo1(1044-1559) was cut with BamHI/EcoRI was ligated into pGEX6P1 that contains a precision protease cleavage site between the GST and $M L D$ reading frames. To create an MLD construct C-terminally tagged with (myc) 6 for expression in yeast, a fragment consisting of Myo1 promoter-Myo1(1044-1569) was cut from pNT49 using PstI and BamHI and ligated into pRL72 cut PstI/BamHI resulting in pIL13.

\section{Quantification of Myo I-GFP and deletion construct bud neck localization}

Exponentially growing cultures of cells expressing either Myo1-GFP or a deletion construct were assessed for bud neck localization using an Eclipse E800 microscope with a 100/1.40 oil differential-interference contrast objective (Nikon, Melville, NY). At least 100-200 live cells were analyzed, and data shown are averages from at least 3 independent experiments. Images were collected with a $0.5 \mathrm{~s}$ exposure to fluorescent light filtered through an EXHQ450/50 DM480 LP/BA465LP GFP filter set (Chroma, Brattleboro, VT) with a cooled RTE/CCD 782Y Interline camera (Princeton Instruments) using MetaMorph (Universal Imaging Corp., West Chester, PA). RLY2127 and 2128 fluorescence was weak and could not be collected using the method described above. Instead 20 $\mathrm{z}$ sections at $0.5 \mu \mathrm{m}$ intervals were collected and deconvolved as described below for the CR movies. RLY1044 and RLY1046 were also measured in this way to ensure that counts were comparable with those obtained by the first method (Results for RLY1044: $79 \%$. Results for RLY1046: 75 \%).

\section{Fluorescence recovery after photobleaching (FRAP)}

FRAP experiments were carried out using a $435 \mathrm{~nm}$ nitrogen pulsed Micropoint laser (Photonic Instruments Inc., St. Charles IL). Galvanometer based steering optics controlled with MetaMorph software were used to position the laser beam, which was focused into a diffraction-limited $0.7 \mu \mathrm{m}$ spot using a Nikon $100 \times 1.4$ N.A. objective lens. Images were acquired every 5 or $10 \mathrm{~s}$ with a Yokogawa spinning disc confocal microscope mounted on a Nikon TE2000U stand and a Hamamatsu ORCA ER cooled CCD. All measurements were done using MetaMorph and all data analysis was done using Excel (Microsoft). To analyse the data, the signal from the FRAPed bud neck and a control bud neck (to check for bleaching) were collected. Regression analysis to determine the half time to maximum was done using a one-phase exponential association function $(\mathrm{Y}=$ bottom + (top-bottom) $\cdot(1$-exp $[-\mathrm{k} \cdot \mathrm{x}])$, where $\mathrm{k}$ is the rate constant and $t 1 / 2$ is $0.69 / \mathrm{k}$ in Prism (version 4.00; GraphPad).

\section{Time-lapse imaging of $C R$ contraction}

For assaying CR contraction, cells were arrested in metaphase with nocodazole for $3 \mathrm{hrs}$ at room temperature and then released from the arrest. The released cells were mounted on gelatine pads. Three dimensional (3D) images and movies of RLY1331 and RLY2139 were collected with the spinning disc confocal microscope described above, imaging 12 sections in the z-plane with a spacing of $0.5 \mu \mathrm{m}$, with exposures of $1 \mathrm{~s}$. Each stack of zseries images was deconvolved and rendered in to $3 \mathrm{D}$ images and movies using MetaMorph (Universal Imaging Corp., West Chester, PA).

\section{Protein purification}

Myo1 minimum localisation domain (MLD) tagged Nterminally with GST (plasmid pIL10) was transformed into BL2 1 cells and grown in $1.5 \mathrm{~L}$ cultures at $37^{\circ} \mathrm{C}$ until an $\mathrm{OD}_{600}$ of 1-1.2. The temperature was reduced to $24^{\circ} \mathrm{C}$ and IPTG was added to a final concentration of $50 \mu \mathrm{M}$ before growing the cultures for a further 4 hrs. The cells were harvested and stored as pellets at $-20^{\circ} \mathrm{C}$. Pellets were lysed in $25 \%$ sucrose, $50 \mathrm{mM}$ Tris $\mathrm{HCl}, \mathrm{pH} 7.5,500 \mathrm{mM}$ $\mathrm{NaCl}, 1$ Roche inhibitor tablet per $50 \mathrm{ml}$ solution, $2 \mathrm{mM}$ DTT. The lysate was centrifuged at 35,000 rpm for $30 \mathrm{~min}-$ utes and the supernatant added to $800 \mu$ l Glutathione Sepharose 4B (Amersham Biosciences) for 3 hrs at $4{ }^{\circ} \mathrm{C}$ with constant agitation. The resin was washed with $50 \mathrm{ml}$ buffer A+ Triton ( $500 \mathrm{mM} \mathrm{NaCl}, 50 \mathrm{mM}$ Tris $\mathrm{HCl} \mathrm{pH} \mathrm{7.5,}$ $1 \mathrm{mM}$ DTT, $1 \%$ Triton X-100) followed by $40 \mathrm{ml}$ buffer A (500 mM NaCl, $50 \mathrm{mM}$ Tris $\mathrm{HCl}$ pH 7.5, $1 \mathrm{mM}$ DTT). The resin was resuspended in 300-500 $\mu \mathrm{l}$ buffer A and $30 \mu \mathrm{l}$ of GST tagged precision protease at $0.6 \mathrm{mg} / \mathrm{ml}$ was added. Digestion was carried out overnight at $4{ }^{\circ} \mathrm{C}$ and the supernatant was removed after low speed centrifugation to pellet the resin.

\section{Gel filtration analysis}

A Superose 6 column (BioRad) was equilibrated in buffer A. $250 \mu \mathrm{l}$ of Glutathione sepharose purified MLD with the GST tag removed was added and $0.5 \mathrm{ml}$ fractions were collected. Alternatively, $250 \mu \mathrm{l}$ RLY2132 yeast grinding extract (see below) was run over the column. For SDSPAGE analysis, $10 \%$ TCA was added to each fraction, incubated on ice for 30 minutes and centrifuged for 15 minutes. The pellets were resuspended in $30 \mu \mathrm{l} \mathrm{SDS-load-}$ ing buffer and $5 \mu \mathrm{l} 1 \mathrm{M}$ non-pH'd Tris base. MLD-(myc)6 from yeast extract fractions run on the column was detected by Western blotting using the anti-myc mono- 
clonal antibodies, 9E-10. The column was calibrated with globular proteins of known Stokes radii: Thyroglobulin $669 \mathrm{kDa}, 8.5 \mathrm{~nm}$, Ferritin - $440 \mathrm{kDa}, 6.1 \mathrm{~nm}$, Catalase $232 \mathrm{kDa}, 5.2 \mathrm{~nm}$, Aldolase $158 \mathrm{kDa}, 4.8 \mathrm{~nm}$. Dextran Blue with a molecular weight of approximately $2000 \mathrm{kDa}$ was used to establish the void volume of the column.

\section{Sucrose density centrifugation gradients}

$5 \mathrm{ml} \mathrm{6-20 \%} \mathrm{sucrose} \mathrm{gradients} \mathrm{were} \mathrm{prepared} \mathrm{in} \mathrm{ultra} \mathrm{clear}$ centrifuge tubes (Beckman) for the SWTi55 rotor (Beckman). The gradient was calibrated using protein standards of known sedimentation coefficients: catalase - $232 \mathrm{kDa}$, $11.2 \mathrm{~S}$, aldolase - $158 \mathrm{kDa}, 7.3 \mathrm{~S}$, BSA - $66 \mathrm{kDa}, 4.6 \mathrm{~S}$, ovalbumin $-43 \mathrm{kDa}, 3.6 \mathrm{~S}$. The top $400 \mu \mathrm{l}$ of the gradient were removed and replaced with $300 \mu \mathrm{l}$ Glutathione sepharose 4B purified MLD, $5 \mu \mathrm{g}$ of aldolase as an internal control, sucrose to a final concentration of $6 \%$ and water to $400 \mu \mathrm{l}$. The gradient was centrifuged for $19 \mathrm{hrs}$ at $40 \mathrm{k}$ $\mathrm{rpm}$ at $4{ }^{\circ} \mathrm{C}$. At the end of the run, the gradient was fractionated from the top into $250 \mu \mathrm{l}$ fractions. These were TCA precipitated as described above.

\section{Calculation of native molecular weight}

The native molecular weight of the expressed MLD was calculated using its Stokes radius measured by gel filtration and its sedimentation coefficient determined by sucrose density gradient centrifugation using the following equation 1 described in [31].

$$
\mathrm{M} \cdot \mathrm{W}=\frac{S \omega_{20} N \cdot 6 \pi \cdot \mathrm{n} \cdot \mathrm{R}_{\mathrm{s}}}{1-\overline{v \rho}}
$$

where $S \omega_{20}$ is the sedimentation coefficient, $R_{s}$ is the Stokes radius, Avogadro's number, $N$ is $6.02 \times 10^{23}$; viscosity coefficient, $\mathrm{n}$ is $1 \times 10^{-2} \mathrm{~g} \mathrm{~s}^{-1} \mathrm{~cm}^{-1}$; solution density, $\rho$ is $1 \mathrm{~g} \mathrm{~cm}^{-3}$ and partial specific volume, $\bar{v}$ is $0.71 \mathrm{~cm}^{3} \mathrm{~g}^{-1}$ (assumed to be that of an average soluble protein).

\section{Yeast extracts}

To prepare the yeast extracts used for gel filtration analysis, small pellets were extruded through a syringe into $\mathrm{N}_{2}$ (l) creating cylindrical fragments that could then be ground by pestle and mortar in the presence of $\mathrm{N}_{2}(\mathrm{l})$. The powdered yeast was thawed and resuspended in buffer $\mathrm{A}$. Protease inhibitors $(2 \mu \mathrm{g} / \mathrm{ml}$ Antipain, $2 \mu \mathrm{g} / \mathrm{ml}$ Leupeptin, $2 \mu \mathrm{g} / \mathrm{ml}$ Aprotinin, $2 \mu \mathrm{g} / \mathrm{ml}$ Pepstatin, $2 \mu \mathrm{g} / \mathrm{ml}$ Chymostatin) and $1 \mathrm{mM}$ PMSF were added to both buffers. Lysis was checked using light microscopy. Thawed extracts were centrifuged at $50 \mathrm{~K} \mathrm{rpm}$ in a Sorvall RP120AT rotor for 30 minutes at $4{ }^{\circ} \mathrm{C}$.

\section{Authors' contributions}

NT created the plasmids and strains and performed the experiments to assess bud neck localization of the differ- ent Myo1 constructs, with the exception of pIL11 and RLY2127 and RLY 2128. Other strains containing pIL11 were created by IMBL. IMBL performed all other experiments and drafted the manuscript. RL conceived of the study, participated in its design and coordination. IMBL, NT and RL revised the manuscript. All authors read and approved the final manuscript.

\section{Acknowledgements}

The authors would like to thank HMS Nikon Imaging Centre Director J.Waters, HMS Nikon Imaging Centre Technician L. Petrak and P. Marina and R. Wedlich-Soldner for technical discussion of the manuscript, and Dorit Hanein and Neils Volkmann for valuable discussion on coiled coil structures. This work was funded by NIH grant GM59964.

\section{References}

I. Tolliday N, Pitcher M, Li R: Direct evidence for a critical role of myosin II in budding yeast cytokinesis and the evolvability of new cytokinetic mechanisms in the absence of myosin II. Mol Biol Cell 2003, I 4:798-809.

2. Bi E, Maddox P, Lew DJ, Salmon ED, McMillan JN, Yeh E, Pringle JR: Involvement of an actomyosin contractile ring in Saccharomyces cerevisiae cytokinesis. J Cell Biol I998, I 42: I30 I-I2.

3. Luo J, Vallen EA, Dravis C, Tcheperegine SE, Drees B, Bi E: Identification and functional analysis of the essential and regulatory light chains of the only type II myosin Myo I p in Saccharomyces cerevisiae. J Cell Biol 2004, I 65:843-55.

4. Boyne JR, Yosuf HM, Bieganowski P, Brenner C, Price C: Yeast myosin light chain, MIclp, interacts with both IQGAP and class II myosin to effect cytokinesis. J Cell Sci 2000, I I3(Pt 24):4533-43.

5. Tolliday N, Bouquin N, Li R: Assembly and regulation of the cytokinetic apparatus in budding yeast. Curr Opin Microbiol 200I, 4:690-5.

6. Sellers JR: Myosins: a diverse superfamily. Biochim Biophys Acta 2000, 1496:3-22.

7. Tan JL, Ravid S, Spudich JA: Control of nonmuscle myosins by phosphorylation. Annu Rev Biochem 1992, 6 I:721-59.

8. Motegi F, Mishra M, Balasubramanian MK, Mabuchi I: Myosin-II reorganization during mitosis is controlled temporally by its dephosphorylation and spatially by Mid I in fission yeast. J Cell Biol 2004, I 65:685-95.

9. Hostetter D, Rice S, Dean S, Altman D, McMahon PM, Sutton S, Tripathy A, Spudich JA: Dictyostelium myosin bipolar thick filament formation: importance of charge and specific domains of the myosin rod. PLoS Biol 2004, 2:e356.

10. Sabry JH, Moores SL, Ryan S, Zang JH, Spudich JA: Myosin heavy chain phosphorylation sites regulate myosin localization during cytokinesis in live cells. Mol Biol Cell 1997, 8:2605-I5.

II. Nakasawa T, Takahashi M, Matsuzawa F, Aikawa S, Togashi Y, Saitoh $T$, Yamagishi A, Yazawa M: Critical regions for assembly of vertebrate nonmuscle myosin II. Biochemistry 2005, 44: I74-83.

12. Bezanilla M, Pollard TD: Myosin-II tails confer unique functions in Schizosaccharomyces pombe: characterization of a novel myosin-II tail. Mol Biol Cell 2000, I I:79-9I.

13. Mulvihill DP, Barretto C, Hyams JS: Localization of fission yeast type II myosin, Myo2, to the cytokinetic actin ring is regulated by phosphorylation of a C-terminal coiled-coil domain and requires a functional septation initiation network. $\mathrm{Mol}$ Biol Cell 200I, I 2:4044-53.

14. McCollum D, Feoktistova A, Gould KL: Phosphorylation of the myosin-II light chain does not regulate the timing of cytokinesis in fission yeast. J Biol Chem 1999, 274:|769|-5.

15. Berger B, Wilson DB, Wolf E, Tonchev T, Milla M, Kim PS: Predicting coiled coils by use of pairwise residue correlations. Proc Natl Acad Sci U S A 1995, 92:8259-63.

16. Gruber M, Lupas AN: Historical review: another 50th anniversary - new periodicities in coiled coils. Trends Biochem Sci 2003, 28:679-85.

17. Chen J, Skehel J], Wiley DC: $\mathbf{N}$ - and $\mathbf{C}$-terminal residues combine in the fusion-pH influenza hemagglutinin $\mathrm{HA(2)}$ subunit 
to form an $\mathbf{N}$ cap that terminates the triple-stranded coiled coil. Proc Natl Acad Sci U S A 1999, 96:8967-72.

18. Boudko SP, Strelkov SV, Engel J, Stetefeld J: Design and crystal structure of bacteriophage T4 mini-fibritin NCCF. J Mol Biol 2004, 339:927-35.

19. Peter BJ, Kent HM, Mills IG, Vallis Y, Butler PJ, Evans PR, McMahon HT: BAR domains as sensors of membrane curvature: the amphiphysin BAR structure. Science 2004, 303:495-9.

20. Tai LJ, McFall SM, Huang K, Demeler B, Fox SG, Brubaker K, Radhakrishnan I, Morimoto RI: Structure-function analysis of the heat shock factor-binding protein reveals a protein composed solely of a highly conserved and dynamic coiled-coil trimerization domain. J Biol Chem 2002, 277:735-45.

21. Kammerer RA, Kostrewa D, Progias P, Honnappa S, Avila D, Lustig A, Winkler FK, Pieters J, Steinmetz MO: A conserved trimerization motif controls the topology of short coiled coils. Proc Natl Acad Sci U S A 2005, 102:13891-6.

22. Lupas A, Van Dyke M, Stock J: Predicting coiled coils from protein sequences. Science 1991, 252:1 162-4.

23. Lupas A: Prediction and analysis of coiled-coil structures. Methods Enzymol 1996, 266:513-25.

24. Lord M, Laves E, Pollard TD: Cytokinesis Depends on the Motor Domains of Myosin-II in Fission Yeast but Not in Budding Yeast. Mol Biol Cell 2005, 16:5346-55.

25. Schmidt M, Bowers B, Varma A, Roh DH, Cabib E: In budding yeast, contraction of the actomyosin ring and formation of the primary septum at cytokinesis depend on each other. J Cell Sci 2002, II 5:293-302.

26. VerPlank L, Li R: Cell cycle-regulated trafficking of Chs2 controls actomyosin ring stability during cytokinesis. Mol Biol Cell 2005, 16:2529-43

27. Negron JA, Gonzalez S, Rodriguez-Medina JR: In vivo phosphorylation of type II myosin in Saccharomyces cerevisiae. Biochem Biophys Res Commun 1996, 22 I:5I5-20.

28. Sherman F, Fink G, Lawrence C: Methods in Yeast Genetics. Cold Spring Harbor, New York: Cold Spring Harbor Laboratory Press;; 1974.

29. Kaiser C, Michaelis S, Mitchell A: Methods in yeast genetics: a laboratory course manual. Cold Spring Harbor, New York.: Cold Spring Harbor Laboratory Press;; 1994.

30. Lippincott J, Li R: Sequential assembly of myosin II, an IQGAP. like protein, and filamentous actin to a ring structure involved in budding yeast cytokinesis. J Cell Biol 1998, 1 40:355-66.

31. Post PL, Tyska MJ, O'Connell CB, Johung K, Hayward A, Mooseker MS: Myosin-IXb is a single-headed and processive motor. J Biol Chem 2002, 277: I1679-83.
Publish with Bio Med Central and every scientist can read your work free of charge

"BioMed Central will be the most significant development for disseminating the results of biomedical research in our lifetime. "

Sir Paul Nurse, Cancer Research UK

Your research papers will be:

- available free of charge to the entire biomedical community

- peer reviewed and published immediately upon acceptance

- cited in PubMed and archived on PubMed Central

- yours - you keep the copyright
BioMedcentral 